\title{
Linoleic acid and risk of sudden cardiac death
}

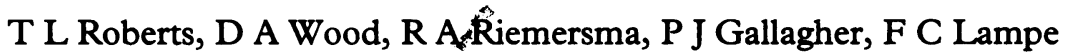<smiles>[3H][13CH3]</smiles>

Abstract

Objective-To test the hypothesis that the essential fatty acid, linoleic acid, measured in adipose tissue as an indicator of long term dietary intake, is inversely related to the risk of sudden cardiac death.

Design-A population case-control study. Setting-A regional health district.

Subjects-84 men (age 25-64 years) who died instantaneously or within 24 hours of the onset of symptoms with no history of coronary heart disease or medically treated hyperlipidaemia, and in whom coronary artery disease was diagnosed at postmortem examination as the primary cause of death, were compared with 292 age (to within two years) and sex matched healthy controls and their partners drawn from the general practitioners' records with whom the cases were registered.

Main outcome measures-Fatty acid composition of adipose tissue and the risk of sudden cardiac death.

Results-Linoleic acid in adipose tissue was inversely related to the risk of sudden cardiac death. The estimated relative risk $(95 \% \mathrm{CI})$ of sudden cardiac death was $5.7(1.8$ to 17.9$)$ for the lowest quintile and $4.0(1.2$ to 12.9$)$ for the next quintile of adipose linoleic acid in the control population when compared with the highest quintile. This inverse relation was independent of age, reported smoking habits, history of hypertension, and diabetes, although there was a close association with cigarette smoking. The estimated adjusted proportionate increase in risk $(95 \% \mathrm{CI})$ of sudden cardiac death was $1.14(1.03$ to 1.23$)$ for every $1 \%$ reduction of linoleic acid in adipose tissue.

Conclusions-The percentage content of linoleic acid in adipose tissue was inversely related to the risk of sudden cardiac death. Populations with a high risk of sudden cardiac death may benefit from increasing their dietary intake of polyunsaturated fatty acid oils, principally from cereals and vegetables.

\section{(Br Heart f 1993;70:524-529)}

Sinclair first put forward the hypothesis that a chronic relative deficiency of polyenoic essential fatty acids, in particular linoleic acid, was a cause of coronary thrombosis. ${ }^{1}$ Linoleic acid is essential because it cannot be synthesised by humans; the only source is our diet. We have investigated the relation between linoleic acid, measured in adipose tissue as an indicator of long term dietary intake, and the risk of coronary heart disease in several population studies. $^{2}$ Cross cultural comparisons of healthy middle aged men in populations with different coronary mortality-namely, Finland, Scotland, Sweden, and Italy-have consistently shown lower frequency distributions of this fatty acid in countries with a high mortality, ${ }^{34}$ and a between population study of middle aged men and women in Scotland has also shown an inverse correlation between linoleic acid in adipose tissue and standardised mortality ratios for coronary heart disease in different districts. ${ }^{5}$ An inverse relation between adipose linoleic acid and the risk of acute myocardial infarction and angina pectoris has also been found for men with a threefold increase in risk for each of these non-fatal manifestations of the disease in the lowest quintile of distribution of linoleic acid within populations. ${ }^{67}$ These inverse relations are independent of the major risk factors for coronary heart disease although there was a close association with smoking as cigarette smokers eat significantly less linoleic acid and had correspondingly lower proportionate storage of this fatty acid compared with nonsmokers. $^{7}$ We now report the relation between linoleic, and other fatty acids, and the risk of sudden cardiac death.

\section{Patients and methods}

SELECTION OF CASES OF SUDDEN CARDIAC DEATH

All men aged 25-64 years resident in the Southampton and South West Hampshire Health District (population in 1990 was 104457 in this age and sex group) who died instantaneously or within 24 hours of the onset of symptoms were considered. Those with no history of coronary heart disease (diagnosis of angina, myocardial infarction, or coronary artery revascularisation) or medically treated hyperlipidaemia were eligible for the study, if coronary artery disease was diagnosed as the primary cause of death at postmortem examination. With the assistance of HM Coroner's office and the Department of Pathology, Southampton University Hospitals, which together are responsible for all coroners' postmortem examinations, community deaths were monitored and all cases of incident sudden cardiac death identified. 
Cases were also identified through surveillance of the accident and emergency department and medical wards at Southampton General Hospital and the coronary care unit and medical wards at the Royal South Hampshire Hospital for deaths in hospital.

The 84 cases of sudden cardiac death that met the criteria were identified between 1 January 1990 and 31 July 1991 and in 66 (78.6\%) adipose tissue samples were taken from the anterior abdominal wall at postmortem examinations and until analysis stored at $-70^{\circ} \mathrm{C}$. In 14 cases samples were missed and in four cases the bodies were severely decomposed.

The coroner's officer interviewed a relative, bystander, or neighbour in $81(96.4 \%)$ cases and a relative was identified in $78(92.9 \%)$, from whom he obtained the information on name, date of birth, marital state, occupation, and address of the deceased, together with place, time, and circumstances of death, his medical history, and medication. The general practitioner with whom the deceased was registered was approached and permission sought to interview the nearest surviving relative. Of the 78 relatives 64 were married partners, five were divorcees, and nine blood relatives (two mothers, five siblings, and two daughters). The $40(51 \cdot 3 \%)$ relatives or other contacts interviewed were 33 married partners, one mother, four siblings, and two daughters. Two others were interviewed. They were a long-term friend and a landlady of one of the deceased. Twenty six relatives refused interviews or could not be contacted. In 12 cases the general practitioner refused permission to approach the relative. Information was obtained on the deceased's medical history, smoking habit, alcohol consumption, and family history of heart disease.

\section{SELECTION OF CONTROLS}

Controls were selected from the Hampshire Family Practitioner Committee's computerised patient register. For each case of sudden cardiac death four male controls frequency matched for age (within two years) were drawn from the records of the general practitioner with whom the case was registered with the object of interviewing two controls for every case. The 711 controls selected were checked by their general practitioner and after 25 medical exclusions for psychotic or terminal illness. 686 were contacted by letter and invited, together with their partner, to the preventive cardiology clinic (Royal South Hampshire Hospital) for a health check. Fifty one letters were returned by the Post Office as "not known at this address", 61 refused, and 224 did not attend. Of the 358 (response rate $52 \%$ ) male controls that were seen, 292 $(82.0 \%)$ attended with their married partners. To validate the female partner's report of the man's demographic details, medical history, smoking habit, alcohol consumption, and family history of heart disease, male controls and their partners were interviewed independently by the same physician (TLR). In the men, height, weight, and waist and hip cir- cumference were measured while they were lightly clothed. Sitting blood pressure was measured in the left arm after five minutes rest with diastolic pressure measured at phase $\mathrm{V}$ (Hawksley random zero sphygmomanometer). A non-fasting sample of blood was taken by vacutainer, centrifuged, and aliquots of serum and plasma were stored at $-70^{\circ} \mathrm{C}$. Adipose tissue was sampled from 292 $(82 \cdot 0 \%)$ male controls under local anaesthetic ( $2 \%$ lignocaine) from the anterior abdominal wall with syringe suction through a 16 gauge needle, washed in a freshly prepared $1 \%$ solution of vitamin $C$ in normal saline, and stored at $-70^{\circ} \mathrm{C}$. Thirty two controls refused to give a sample. Thirty subjects reported a history of coronary heart disease at the health check and were therefore not asked to give a sample. Therefore, 230 (64.6\%) male controls had adipose tissue sampled and questionnaires were answered by themselves and their wives.

\section{LABORATORY PROCEDURES}

All laboratory analyses were performed by the cardiovascular research unit and the staff were not aware whether the samples were from cases or controls.

The samples of adipose tissue were thawed, rinsed in saline, and the lipids were extracted into distilled heptane. This extract was then washed with isopropanol $(0.05 \%)$ in potassium hydroxide (w/v) to remove nonesterfied fatty acids and phospholipids. The neutral lipid extract was reduced to dryness under vacuum and dissolved in toluene. Fatty acid methyl esters were prepared by direct transmethylation (10 minutes at $50^{\circ} \mathrm{C}$ with $0.5 \mathrm{M}$ sodium methoxide in methanol). The esters were washed with acidified water, and re-extracted into hexane, and the hexane layer evaporated under vacuum. The methyl esters were redissolved in $40 \mu \mathrm{l}$ redistilled choloroform for analysis on a Pye Chromatograph 204. This was fitted with a $1.5 \mathrm{~m}$ column packed with GP $10 \%$ SP-2330 on 100/120 mesh Chromosorb WAW (Supelco). The peaks were quantified with a flame ionisation detector and Perkin Elmer Nelson integrator. The retention times of authentic fatty acid methyl esters were used for identification.

\section{STATISTICAL ANALYSIS}

The mean proportion of linoleic acid in adipose tissue in men from our previous surveys allowed us to estimate that 75 cases and 150 controls would be required to detect a difference of 0.34 of a standard deviation at the $p<0.05$ level of significance in a two sided test with $80 \%$ power.

Comparisons between cases and controls were made with non-parametric tests. The Mann-Whitney U test was used for comparisons of continuous variables and the $\chi^{2}$ (with continuity correction) for categorical data. A logistic regression was used to assess the independent contribution of linoleic acid in adipose tissue and other risk factors of coronary heart disease to the risk of sudden cardiac 
death, in a stepwise procedure, with a cut off of $p=0 \cdot 05$. The significance of each factor included in the model was tested by means of the likelihood ratio statistic. Adjusted odds ratios with $95 \%$ confidence intervals $(95 \%$ CIs) were calculated. The analysis was carried out with the logistic regression procedure in SPSS v4 on an IBM 3090 mainframe computer.

The data from risk factors of coronary heart disease used in the regression analyses were obtained from the relatives of controls. Agreement between responses of controls and their spouses was tested with the Kappa statistic. For the cases, information from relatives and coroners officers was used, or from 'coroners' officers only when no relatives were interviewed. The records of the coroners' officers were incomplete for smoking habit, but six cases were confirmed as smokers in addition to those confirmed by relatives.

The study was approved by the Southampton and South West Hampshire District Health Authority Ethics Committee (submission number 103/87).

\section{Results}

CASES OF SUDDEN CARDIAC DEATH

The 84 cases of sudden cardiac death identified had a mean age of 54.9 (range 33-64) years and $68(80.9 \%)$ died in the community, 55 at home, 16 in hospital, and 13 elsewhere (six at work, four in a public place, two in private cars and one at another person's home). Deaths showed no temporal pattern by time of day, day of the week, and month of the year. Thirty nine $(46.4 \%)$ deaths were witnessed and in $13(33.3 \%)$ death was instantaneous. For the 42 cases of sudden cardiac death where relatives were interviewed 33 (78.5\%) had one or more symptoms before death and $26(61.9 \%)$ of these had chest pain. Twenty two $(52.4 \%)$ were taking one or more drugs before death. Table 1 shows the pathological findings at postmortem examination.

Cases of sudden cardiac death with and without adipose samples did not differ significantly in age, time of day, day of the week or month of death, place of death, and whether the death was witnessed or not. Nor was the

Table 1 Postmortem examination results in 84 cases of sudden cardiac death

\begin{tabular}{lc}
\hline & No (\%) \\
\hline $\begin{array}{l}\text { Severity of coronary artery disease } \\
\text { Mild }\end{array}$ & $3(3 \cdot 6)$ \\
Moderate & $21(25 \cdot 0)$ \\
Severe & $59(70 \cdot 2)$ \\
Coronary artery thrombus & $35(41 \cdot 7)$ \\
Macroscopic evidence of: & \\
Acute infarction & $27(32 \cdot 1)$ \\
Old infarction & $22(26 \cdot 2)$ \\
Left ventricular: &. \\
Hypertrophy & $45(53 \cdot 6)$ \\
Dilatation & $7(8 \cdot 3)$ \\
Rupture & $3(3 \cdot 6)$ \\
Mean heart weight (g) (range) & $438(286-740)$ \\
\hline * case not classifiable. &
\end{tabular}

proportion with relatives identified and interviewed different. Smoking habit, diabetes, alcohol consumption, medication, and family history of premature ( $<60$ years) coronary heart disease did not differ between those with and without samples but a history of hypertension was significantly more common in those who were not sampled. Heart weight, prevalence of thrombus, acute infarction, old infarction, and left ventricular hypertrophy were not significantly different between the groups.

\section{ADIPOSE FATTY ACID COMPOSITION}

Cases of sudden cardiac death had significantly lower linoleic acid (C18:2) compared with controls (table 2). Cases also had significantly lower palmitic (C16:0), and higher palmitoleic (C16:1), oleic (C18:1), gondoic (C20:1), and eicosadienoic (C20:2n-6) acid compared with controls. Total saturated and polyunsatured fatty acids were significantly lower in cases, and monounsaturated acids significantly higher in cases, but there was no difference between cases and controls in the ratio of polyunsaturated: saturated fatty acids.

\section{RISK FACTORS REPORTED BY PARTNERS}

Agreement between male controls and their married partners on the man's smoking habit (Kappa 0.88), medically treated hypertension (Kappa 0.92), and diabetes (Kappa 0.85) was stron'g with only a small number of misclassifictions.

Reported cigarette smoking by the partners of the victims of sudden cardiac death was significantly more prevalent than by control partners (table 3). Ex-cigarette smokers and lifelong non-smokers were also more common in the controls. A family history of premature heart disease ( $<60$ years) in a first degree relative was three times more likely in victims of sudden cardiac death, but there was no difference in the proportion of either medically treated hypertension or diabetes between cases and controls.

\section{LINOLEIC ACID AND THE ESTIMATED RELATIVE} RISK OF SUDDEN CARDIAC DEATH

The contribution of adipose linoleic acid to the risk of sudden cardiac death was estimated with stepwise logistic regression. With sudden cardiac death as the dependent variable the analysis used age, current cigarette smoking reported by relatives or coroner's officer, history of treated hypertension, history of treated diabetes, and adipose linoleic acid in 65 cases and 230 controls with complete data. Linoleic acid $\left(\chi^{2}=9.11 ; p=0.03\right)$ made a significant and independent contribution to the explanation of sudden cardiac death together with smoking habit.

Table 4 shows the estimated unadjusted and adjusted relative risks and $95 \%$ CIs of sudden cardiac death by quintiles of distribution of adipose linoleic acid in the control population. The estimated unadjusted proportionate increase in risk $(95 \% \mathrm{CI})$ of sudden cardiac death was $1.16(1.06$ to 1.27$)$, and after adjustment for the above coronary 
Table 2 Mean percentage (SEM) of adipose tissue fatty acids in healthy controls and cases of sudden cardiac death

\begin{tabular}{|c|c|c|c|}
\hline & Fatty acidst & $\begin{array}{l}\text { Sudden cardiac } \\
\text { death }(n=66)\end{array}$ & $\begin{array}{l}\text { Controls } \\
(n=292)\end{array}$ \\
\hline $\begin{array}{l}\text { Saturated: } \\
\text { Myristic } \\
\text { Palmitic } \\
\text { Stearic } \\
\text { Total }\end{array}$ & $\begin{array}{l}\text { C14:0 } \\
\text { C16:0 } \\
\text { C18:0 }\end{array}$ & $\begin{array}{c}3.35(0 \cdot 10) \\
21 \cdot 5^{\star \star}(0 \cdot 28) \\
4 \cdot 65(0 \cdot 16) \\
29 \cdot 5^{\star \star}(0.45)\end{array}$ & $\begin{array}{c}3.30(0.04) \\
22 \cdot 1(0.12) \\
4 \cdot 89(0.06) \\
30.3(0.17)\end{array}$ \\
\hline $\begin{array}{l}\text { Monounsaturated: } \\
\text { Palmitoleic } \\
\text { Oleic } \\
\text { Gondoic } \\
\text { Total }\end{array}$ & $\begin{array}{l}\text { C16:1n-7 } \\
\text { C18:1n-9 } \\
\text { C20:1n-9 }\end{array}$ & $\begin{array}{c}6 \cdot 79^{\star \star}(0 \cdot 24) \\
46 \cdot 9^{\star \star}(0 \cdot 30) \\
2 \cdot 30^{\star \star}(0 \cdot 08) \\
56 \cdot 0^{\star \star}(0 \cdot 48)\end{array}$ & $\begin{array}{c}5.95(0.09) \\
45.9(0.14) \\
2.04(0.03) \\
53.9(0.20)\end{array}$ \\
\hline $\begin{array}{l}\text { Polyunsaturated: } \\
a \text {-Linolenic } \\
\text { Docosapentaenoic } \\
\text { Docosahexaenoic } \\
\text { Linoleic } \\
\gamma \text {-Linolenic } \\
\text { Eicosadienoic }\end{array}$ & $\begin{array}{l}\text { C18:3n-3‡ } \\
\text { C22:5n-3 } \\
\text { C22:6n-3 } \\
\text { C18:2n-6 } \\
\text { C18:3n-65 } \\
\text { C20:2n-6 }\end{array}$ & $\begin{array}{c}0.73(0.03) \\
0.19(0.01) \\
0.13(0.01) \\
11.2^{\star \star}(0.41) \\
0.32(0.02) \\
0.28^{\star}(0.02)\end{array}$ & $\begin{array}{c}0.76(0.01) \\
0.18(0.003) \\
0.14(0.01) \\
12.1(0.23) \\
0.29(0.01) \\
0.24(0.01)\end{array}$ \\
\hline $\begin{array}{l}\text { Dihomo- } \gamma \\
\text { Linolenic } \\
\text { Arachidonic } \\
\text { Docosatetraenoic } \\
\text { Total }\end{array}$ & $\begin{array}{l}C 20: 3 n-6 \\
C 20: 4 n-6 \\
C 22: 4 n-6\end{array}$ & $\begin{array}{r}0.13(0.01) \\
0.32(0.01) \\
0.09(0.01) \\
13 \cdot 1 \star(0.43)\end{array}$ & $\begin{array}{c}0.13(0.004) \\
0.30(0.01) \\
0.08(0.002) \\
14.5(0.24)\end{array}$ \\
\hline $\begin{array}{l}\text { Ratio of Polyunsaturated: } \\
\text { saturated }\end{array}$ & & $0.45(0.02)$ & $0.49(0.01)$ \\
\hline
\end{tabular}

${ }^{\star} \mathrm{p}<0.05 ;{ }^{\star \star} \mathrm{p}<0.01 ;$ Mann-Whitney $U$ test. †Shorthand notation: $\mathrm{C}$ carbon atoms followed by number of double bonds, and the position of the double bond closest to the terminal methyl carbon atom $(n-x)$. ҒComplex peak including C20:1n-11. §Complex peak including C20:0.

Table 3 Risk factors of coronary heart disease in healthy controls and cases of sudden cardiac death

\begin{tabular}{|c|c|c|}
\hline & $\begin{array}{l}\text { Controls } \\
\text { (from relatives) } \\
(n=292)\end{array}$ & $\begin{array}{l}\text { Cases } \\
\text { (from relatives) } \\
(n=42)\end{array}$ \\
\hline Age (SD) (y) & $57 \cdot 2(6 \cdot 7)$ & $56 \cdot 4(7 \cdot 6)$ \\
\hline $\begin{array}{l}\text { Smoking (\%): } \\
\text { Never smoked } \\
\text { Ex-smoker } \\
\text { Pipe or cigar } \\
\text { Current cigarette }\end{array}$ & $\begin{array}{c}73(25 \cdot 0) \\
139(47 \cdot 6) \\
11(3 \cdot 8) \\
69(23 \cdot 6)\end{array}$ & $\begin{array}{c}4(9 \cdot 5)^{\star} \\
7(16 \cdot 7)^{\star \star} \\
3(7 \cdot 1) \\
28(66 \cdot 7)^{\star \star \star}\end{array}$ \\
\hline $\begin{array}{l}\text { Medical history (\%): } \\
\text { Treated hypertension } \\
\text { Diabetes mellitus } \\
\text { History of heart disease } \\
\text { in first degree relative } \\
<60 \mathrm{yr}(\%)\end{array}$ & $\begin{array}{l}39(13.4) \\
11(3.8)\end{array}$ & $\begin{array}{l}7(16 \cdot 7) \\
1(2 \cdot 4)\end{array}$ \\
\hline
\end{tabular}

heart disease risk factors was 1.14 (1.03 to $1.23)$, for every $1 \%$ reduction in adipose linoleic acid.

With sudden cardiac death as the dependent variable a second regression analysis was performed with age, history of treated hypertension and diabetes, and adipose linoleic acid, but with the assumption that all cases of sudden cardiac death, with smoking information missing $(\mathrm{n}=17)$, were current cigarette smokers. Smoking $\chi^{2}=78 \cdot 3 ; p=0.0001$ ) was the only factor to make a significant and independent contribution to the risk of sudden cardiac death.

\section{Discussion}

The risk of sudden cardiac death in this population is strongly and inversely related to adipose linoleic acid and this is consistent with our previous reports of a similar association with both acute myocardial infarction and angina pectoris. ${ }^{67}$ This inverse relation with sudden cardiac death is independent of those risk factors of coronary heart disease (CAD) that could be measured retrospectively, but is closely associated with smoking cigarettes. Sinclair first postulated a chronic relative deficiency of polyenoic essential fatty acids, of which linoleic acid is the commonest, as a cause of coronary thrombosis. ${ }^{1}$ Clinical studies subsequently reported that patients with atherosclerosis (coronary artery disease, peripheral arterial disease, and cerebrovascular disease) had lower concentrations of linoleic acid in their blood lipid fractions and adipose tissue than controls. ${ }^{8}$

In our cross cultural studies populations with a high coronary mortality (Scotland and Finland) had significantly lower distributions of adipose linoleic acid in middle aged healthy men than countries (Sweden and Italy) with a lower coronary mortality. ${ }^{34}$ Within Scotland there is an inverse relation between mean adipose linoleic acid in men and women and the standardised mortality ratios for CHD in different districts. ${ }^{5}$ The prevalence of CHD among men in a population survey on the east coast of Scotland, diagnosed at the time of examination, was also inversely related to adipose linoleic acid. ${ }^{6}$ Nearly a quarter of all cases of CHD in this study were found in the bottom decile of adipose linoleic acid in the healthy population. A subsequent population case-control study found a threefold risk of angina pectoris and acute myocardial infarction when comparing the lowest and highest quintiles of adipose linoleic acid in the control population, and these inverse relations were independent of the major risk factors of CHD, at least for angina, although there was a close association with smoking, which confounded the relation with myocardial infarction. ${ }^{7}$ Only one prospective population (case-control) study of fatty acids has been reported. In this, Miettinen et al estimated the fatty acid composition of phospholipids, cholesterol esters,

Table 4 Relative unadjusted and adjusted risks (95\% CIs) of sudden cardiac death by adipose linoleic acid composition

\begin{tabular}{|c|c|c|c|c|c|}
\hline & \multicolumn{5}{|c|}{ Quintities \% of linoleic acid } \\
\hline & $1(<9 \cdot 5)$ & $2(9.5<11.5)$ & $3(11 \cdot 5<13.0)$ & $4(13 \cdot 0<15.7)$ & $5(15 \cdot 7)$ \\
\hline $\begin{array}{l}\text { Cases }(n=65) \\
\text { Controls }(n=230) \\
\text { Unadjusted odds ratio } \\
\text { (95\% CI) } \\
\text { Adjusted† odds ratio }(95 \% \mathrm{CI})\end{array}$ & $\begin{array}{l}23 \\
46 \\
5 \cdot 7 \\
(1 \cdot 8 \text { to } 17 \cdot 9) \\
4 \cdot 4 \\
(1 \cdot 4 \text { to } 14 \cdot 1)\end{array}$ & $\begin{array}{l}16 \\
46 \\
4.0 \\
(1.2 \text { to } 12 \cdot 9) \\
3.5 \\
(1.1 \text { to } 11 \cdot 5)\end{array}$ & $\begin{array}{l}10 \\
46 \\
2.5 \\
(0.7 \text { to } 8 \cdot 5) \\
2 \cdot 4 \\
(0.7 \text { to } 8 \cdot 3)\end{array}$ & $\begin{array}{l}12 \\
46 \\
3.0 \\
(0.9 \text { to } 9 \cdot 9) \\
3.0 \\
(0.9 \text { to } 10 \cdot 0)\end{array}$ & $\begin{array}{r}4 \\
46 \\
1 \\
1\end{array}$ \\
\hline
\end{tabular}

†Adjusted for age, current cigarette smoking, history of treatment for hypertension or diabetes. 
and triglycerides from fasting serum. ${ }^{9}$ Cases of non-fatal and fatal myocardial infarction and sudden death had a significantly lower concentration of linoleic acid, and lower total polyunsaturated fatty acids in phospholipids but not cholesterol esters, than in controls. This relation was only significant for those in the lowest quintile of fatty acid distribution but was independent of age, smoking, blood pressure, cholesterol and triglyceride concentrations, obesity, and glucose tolerance.

Two other surveys of populations at necropsy have examined the composition of adipose fatty acids and its relation to death from CHD. Thomas and Scott studied necropsies drawn from 10 areas in England and Wales and found that adipose linoleic acid was almost identical in the cases of death attributed to CHD and to other causes. ${ }^{10}$ In the United States Strong et al compared both black and white men who died from CHD with men who had died from external violence (accident, suicide, or homicide) or any natural cause other than atherosclerotic disease. ${ }^{11}$ Again there was no difference in adipose linoleic acid in those who died from CHD or from other causes, regardless of ethnic origin. Both studies included men with a history of CHD before death, who might have increased their consumption of polyunsaturated fats, thus potentially obscuring a real difference in linoleic acid. In our dietary survey men with a reported diagnosis of CHD (either myocardial infarction or angina pectoris) did have a higher dietary intake of linoleic acid, and a correspondingly higher adipose concentration compared with those in whom we made the diagnosis. ${ }^{6}$ This difference in linoleic acid is consistent with dietary change after diagnosis and is an important conservative bias in retrospective studies. In this study the men had no medical diagnosis of CHD before death and deaths were instantaneous, or within 24 hours of the onset of symptoms. So unlike previous population surveys this potential dietary bias does not exist, and the extent to which the victims of sudden cardiac death changed their diets after a diagnosis of systemic hypertension or diabetes could only underestimate the strength of the relation we have found between linoleic acid and sudden cardiac death.

In animal experiments diets rich in linoleic acid reduce the incidence of serious ventricular arrhythmias induced by coronary ligation in vivo. A strong inverse relation was found between adipose linoleic acid $(r=-0.92)$ and the frequency of ischaemic ventricular fibrillation in rats feeding a diet with polyunsaturated:saturated fatty acid ratio of 2 for only four weeks. ${ }^{12}$ In a longer term experiment the incidence of ventricular tachycardia and fibrillation was significantly lower in rats fed with a supplement of sunflower seed oil for up to 20 months compared with a reference diet. Even after long-term feeding of saturated animal fatty acids the incidence of ventricular fibrillation both on coronary artery occlusion and reperfusion was significantly reduced by supplementing the rats' diet with sunflower seed oil. ${ }^{13}$ This is consistent with linoleic acid having a protective effect against these arrhythmias. ${ }^{14}$ Although there have been no dietary experiments in humans based on a linoleic acid supplement alone, the Oslo primary prevention trial in high risk middle aged men, which used a low saturated high polyunsaturated fatty acid diet, showed a significantly lower incidence of sudden cardiac death in the experimental group. ${ }^{15}$

Although this inverse relation between linoleic acid and sudden cardiac death is consistent with our previous studies of non-fatal manifestations of this disease cautious interpretation is required. There is a strong inverse association between smoking and consumption of linoleic acid and therefore exposure to tobacco could be the real cause and diet a chance association. Alternatively, the relation between smoking cigarettes and CHD, which is largely unexplained except for a higher fibrinogen concentration in smokers, might be partly due to differences in the dietary habits of smokers and non-smokers. Smokers eat less polyunsaturated fatty acids, and in particular less linoleic acid, and this dietary preference, possibly reflecting differences in taste, may be an important determinant of coronary risk in smokers rather than tobacco itself. ${ }^{10}$ It is not possible to separate out these interrelations any further with epidemiological data, but the dietary habits of non-smokers and how these change when someone takes up smoking, or conversely stops the habit, requires investigation. As with any retrospective study fatty acid composition of adipose tissue may reflect changes that result from dying and not the pattern before death, but animal experimental evidence suggests the relative fatty acid distribution remains the same after death for at least 36 hours (R Riemersma, personal communication). Samples of adipose tissue are incomplete among cases and those missed may have a different composition, but given the similarity in other characteristics of those not sampled, this seems unlikely.

The response rate of healthy controls was low and whereas this partly reflects computerised Family Health Service Authority practice lists containing patients who have died, moved away, or are no longer registered, it also represents a true non-response that may introduce bias. Controls who are more middle class and health conscious are likely to have a higher intake of linoleic acid. This could exaggerate the risk of sudden cardiac death associated with lack of this fatty acid but this bias cannot be quantified in our study. Although the response rate of healthy controls is low they are, unlike dead controls, without manifest disease and therefore dietary habits are unlikely to have changed. They were also unselected, except for nonresponse, and therefore give a more representative picture of the distribution of linoleic acid in middle aged men than dead controls.

The measurement of exposure to risk factors of CHD is incomplete for smoking and unobtainable for blood pressure and lipid 
concentrations. If all cases of sudden cardiac death are assumed to be cigarette smokers then tobacco exposure emerges as the only statistically significant factor for sudden death, but even if this assumption were true it does not preclude smoking from operating -through dietary preferences to increase the risk of an arrhythmic death. ${ }^{1314}$ Whether the relation between linoleic acid and sudden cardiac death could be modified by other risk factors in the model is not known, but as blood pressure is only weakly and inversely associated with adipose linoleic acid, and lipids show no strong associations with this fatty acid, ${ }^{7}$ this seems unlikely. In the prospective study of Miettinen et al the relation between linoleic acid in phospholipids and all manifestations of CHD taken together, including death from $\mathrm{CHD}$, was independent of all major risk factors of CHD. 9

Our finding of an inverse relation between adipose linoleic acid and the risk of sudden cardiac death is consistent with that already shown for both acute myocardial infarction and angina pectoris, ${ }^{7}$ and the scientific evidence for causality is strengthened through showing these relations in different populations, at different times, and separately for the major clinical manifestations of CHD..$^{3-7}$ The most likely explanation for these findings is that men who choose diets poor in linoleic acid predispose themselves to the development of angina pectoris, myocardial infarction, and sudden cardiac death. The proportionate storage of linoleic acid in adipose tissue is largely determined by long-term dietary intake, and therefore provides a unique retrospective measure of consumption of this essential fatty acid in victims of sudden cardiac death, although other explanations such as differences in absorption, fatty acid metabolism, and storage of linoleic acid cannot be excluded.

The Committee on Medical Aspects of Food Policy reporting on diet and cardiovascular disease in the United Kingdom recommended a reduction in total fat, and saturated fat in particular, but no specific recommendations were made for polyunsaturated fatty acids. ${ }^{17}$ We believe the epidemiological, clini$\mathrm{cal}$, and experimental evidence of an inverse relation between linoleic acid, the principal polyunsaturated fatty acid, and CHD is now sufficiently strong to recommend that populations with a high mortality from CHD might benefit from supplementing their eating habits with more polyunsaturated oils, principally from cereals and vegetables. Although cigarette smokers are strongly urged to stop, this dietary recommendation is particularly important for those who cannot, especially heavy cigarette smokers, who have the lowest intake of this essential fatty acid.

We thank HM Coroner Mr R N MacKean, coroner's officers, consultant pathologists, and mortuary staff of the Department of Pathology, Southampton University Hospitals, for their help in the identification of cases of sudden cardiac death and collection of samples; Karen Lyall and Jacqui Lawrence for fatty acid analyses; the Hampshire Family Health Services Authority for their help in selecting controls; research nurses in the preventive cardiology clinic: Jenny Cole, Patricia Elliott, Rosemary Ogilvie, Barbara Saunders, and Frances Watts; Christine Bicknell for administration, and all the general practitioners in Southampton district who so willingly eral practitioners in Southampton district who so willingly assisted with this study. TLR had a Merck, Sharp, and Dohme lectureship in preventive cardiology. The study was supported by a grant from the Wellcome Trust $(18907 / 1 \cdot 5)$ and the Preventive Cardiology Trust (administrator, Mr
Dennis Urquhart) that administers the Geoffrey Fryer bequest.

1 Sinclair HM. Deficiency of essential fatty acids and atherosclerosis, etcetra [Letter]. Lancet 1956;1:381-3.

2 Van Staveren WA, Deurenberg P, Katan MB, Burema J, de Groot LCPGM, Hoffmans MDAF. Validity of the fatty acid composition of subcutaneous fat tissue microbiopsies as an estimate of long term average fatty acid biopsies as an estimate of long term average fatty acid
composition of the diet of separate individuals. $\mathrm{Am} \mathcal{F}$

3 Logan RL, Riemersma RA, Thomson M, Oliver MF, Olsson AG, Walldius G, et al. Risk factors for ischaemic heart-disease in normal men aged 40. EdinburghStockholm Study. Lancet 1978;i:949-55.

4 Riemersma RA, Wood DA, Butler S, Elton RA. Oliver MF, Salo $M$, et al. Linoleic acid content in adipose tissue and coronary heart disease. $\mathrm{Br}$ Med $f$ 1986;292:1423-7.

5 Tavendale R, Lee AJ, Smith WCS, Tunstall-Pedoe H. Adipose tissue fatty acids in Scottish men and women: Adipose tissue fatty acids in Scottish men and women:
results from the Scottish Heart Health Study. results from the Scottish
Atherosclerosis 1992;94:161-9.

6 Wood DA, Butler S, Riemersma RA, Thomson M, Oliver MF. Adipose tissue and platelet fatty acids and coronary MF. Adipose tissue and platelet fatty acids and corona

7 Wood DA, Riemersma RA, Butler S, Thomson M, MacIntyre C, Elton R A, et al. Linoleic and eicosapentaenoic acids in adipose tissue and platelets and risk of coronary heart disease. Lancet 1987;i:177-83.

8 Wood D A, Oliver M F. Linoleic acid, antioxidant vitamins and coronary heart disease. In: Marmot M, Elliott P, eds. Coronary heart disease epidemiology: from aetiology to public health. Oxford: Oxford University Press, 1992, (in press).

9 Miettinen T A, Naukkarinen V, Huttenen J K, Mattila S, Kumlin T. Fatty-acid composition of serum lipids predicts myocardial infarction. BMF 1982;285:993-6.

10 Thomas L H, Scott R G. Ischaemic heart disease and the proportions of hydrogenated fat and ruminant-animal at in 1981;35:251-5.

11 Strong J P, Oalmann M C, William $P$ H, Newman W $P$, Tracy R E, Malcolm G T, et al. Coronary heart disease Tracy $R$ E, Malcolm G T, et al. Coronary heart disease in young black and white males in New Orleans: com munity pathology study. Am Heart $f$ 1984;108:747-59.
Sargent C A, Riemersma R A. Polyunsaturated fatty acids and cardiac arrhythmia. Biochemical Society Transactions and cardiac arrhyth

13 McLennan P L, Abeywardena M Y, Charnock J S. Reversal of the arrythymogenic effects of long-term saturated fatty acid intake by dietary $n-3$ and $n-6$ polyunsaturated fatty acids. Am $\mathscr{f}$ Clin Nutr 1990;51:53-8.

14 McLennan P L. Abeywardena M Y, Charnock J S. Influence of dietary lipids on arrhythmias and infarction after coronary ligation in rats. Can $\mathcal{f}$ Physiol Pharmacol 1985;63:1411-7.

15 Hjermann J, Byre K V, Holme I, Leren P. Effect of diet and smoking intervention on the incidence of coronary heart disease. Report from the Oslo Study Group of a randomised trial in healthy men Lancet 1981;ii:1303-10.

16 Thompson R L, Margetts B M, Wood D A, Jackson A A. Cigarette smoking and food and nutrient intakes in relation to coronary heart disease. Nutrition Research Reviews 1992 (in press).

17 Committee on Medical Aspects of Food Policy. Diet and cardiovascular disease. Report of the panel on diet in relation to cardiovascular disease. London: HMSO, 1984. 